\title{
Technological verification of size-optimized 160-channel silicon nitride-based AWG-spectrometer for medical applications
}

\author{
Dana Seyringer $^{1}$ (I) $\cdot$ M. Sagmeister ${ }^{2} \cdot$ A. Maese-Novo ${ }^{3} \cdot$ M. Eggeling ${ }^{3} \cdot$ E. Rank $^{4} \cdot$ P. Muellner ${ }^{3} \cdot$ R. Hainberger ${ }^{3}$. \\ W. Drexler ${ }^{4} \cdot$ M. Vlaskovic ${ }^{2} \cdot$ H. Zimmermann ${ }^{5} \cdot$ G. Meinhardt ${ }^{2} \cdot$ J. Kraft $^{2}$
}

Received: 14 November 2018 / Accepted: 13 April 2019 / Published online: 4 May 2019

(c) The Author(s) 2019

\begin{abstract}
We present the technological verification of a size-optimized 160-channel, 50-GHz silicon nitride-based AWG-spectrometer. The spectrometer was designed for TM-polarized light with a central wavelength of $850 \mathrm{~nm}$ applying our proprietary "AWGParameters" tool. For the simulations of AWG layout, the WDM PHASAR photonics tool from Optiwave was used. The simulated results show satisfying optical properties of the designed AWG-spectrometer. However, the high-channel count causes a large AWG size with standard design approaches. To solve this problem we designed a special taper enabling the reduction of AWG structure by about 15\% while keeping the same optical properties. The AWG design was fabricated and the measured spectra not only confirm the proposed size-reduction but also the improvement of optical properties of the size-optimized AWG.
\end{abstract}

\section{Introduction}

Arrayed Waveguide Gratings (AWGs) are considered an attractive DWDM solution because they represent a compact means of offering higher channel-count technology, have good performance characteristics, and can be more costeffective per channel than other methods [1].

High-index-contrast AWGs, such as Silicon-On-Insulator (SOI) [2] or $\mathrm{Si}_{3} \mathrm{~N}_{4}$-based waveguide devices [3, 4], use a high refractive index contrast material platform. Due to the fact that a waveguide size decreases proportionally to the increase in refractive index contrast, the waveguide size

Dana Seyringer

dana.seyringer@fhv.at

1 Research Centre for Microtechnology, Vorarlberg University of Applied Sciences, Hochschulstr. 1, 6850 Dornbirn, Austria

2 ams AG, Tobelbader Strasse 30, 8141 Premstätten, Austria

3 AIT Austrian Institute of Technology GmbH, Giefinggasse 4, 1210 Vienna, Austria

4 Center for Medical Physics and Biomedical Engineering, Medical University of Vienna, Währinger Gürtel 18-20/4L, 1090 Vienna, Austria

5 Institute of Electrodynamics, Microwave and Circuit Engineering, TU Wien, Gußhausstraße 25/354, 1040 Vienna, Austria for such material compositions shrinks into the sub-micron scale. High-index-contrast makes it possible to guide light in waveguides with a far smaller bending radius, which leads to a significant reduction in the size of AWG structure. Such compact devices can easily be implemented on-chip and have already found applications in WDM systems and also in emerging applications such as optical sensors, devices for DNA diagnostics and optical spectrometers for infrared spectroscopy [5-7].

Arrayed waveguide gratings used as a spectrometer for infrared spectroscopy require a high-channel count (more than one thousand output channels) with a resolution going down to $0.1 \mathrm{~nm}$. However, such high-channel-count AWGspectrometers still feature large size, which can limit their practical use in photonic integrated circuits. To solve this problem, we designed a special taper enabling the reduction of AWG-spectrometer size by about $15 \%$, while maintaining the same optical performance. The technological verification of both AWG designs is also presented.

\section{Optical coherence tomography}

Optical coherence tomography (OCT) is a contact-free imaging method, which has become significantly important in ophthalmology to visualize the retina by measuring the echo of a sample using interferometry. In spectral 
domain OCT (SD-OCT) light from a broad bandwidth light source with low coherence properties is forwarded to an interferometer. Only light that travelled approximately the same distance in both arms of the interferometer will create interference. The interference spectrum is detected using a spectrometer and a line scan camera. The Fourier transformation of the spectrum results in a depth profile (A-scan) and raster scanning over a sample is used to reconstruct a 2D-tomogram (B-scan) or 3D volume (C-scan), showing the cross-sections of the tissue under investigation.

The axial (depth) resolution of a SD-OCT system depends on properties of the light source and is in the range of $5-10 \mu \mathrm{m}$ in commercial systems:

$\Delta z=\frac{2 \ln 2}{\pi}\left(\frac{\lambda c^{2}}{\Delta \lambda}\right)$

with $\lambda c^{2}$, the center wavelength and $\Delta \lambda$, the bandwidth of the light source.

The maximum imaging depth $z_{\max }$, depends on the center wavelength $\lambda c$, and the spectral resolution of the spectrometer and is calculated via:

$z_{\max }=\frac{n_{0} \lambda c^{2}}{4 \delta_{s} \lambda}$

While OCT has massively advanced in terms of resolution and sensitivity in the past $\sim 20$ years, comparably little effort has been taken to reduce size and costs of OCT systems. Commercial OCT systems are usually built with a combination of optical fibers and bulky free spaceoptics, which make such systems sensitive to misalignment resulting in high maintenance costs. Using silicon nitride waveguide-based technologies for OCT applications can make OCT systems more robust, up to five times smaller and up to three times cheaper and, therefore, enable point of care diagnosis.

The goal of the silicon nitride waveguide-based AWG development presented here is to take a major step towards the integration of SD-OCT system operating in a wavelength range of $800-900 \mathrm{~nm}$ and having $0.1 \mathrm{~nm}$ resolution. In the course of the project, key components of an SD-OCT system are being developed and integrated on a single optical waveguide chip employing CMOS compatible processes. Preliminary OCT performance in terms of axial resolution and sensitivity is also presented.

\section{AWG principle}

Arrayed waveguide gratings consist of an array of waveguides (also called phased array, PA) and two star couplers (Fig. 1). One of the input waveguides launches the light consisting of multiple wavelengths $\lambda_{1}-\lambda_{\mathrm{n}}$ into the input star coupler, which then distributes the light amongst an array of waveguides. The light subsequently propagates through the waveguides to the output coupler. The length of these waveguides is chosen such that the optical path length difference between adjacent waveguides $d L$ equals an integer multiple of the central wavelength $\lambda_{c}$ of the demultiplexer. For this wavelength, the fields in the individual arrayed waveguides will arrive at the input of the output coupler with equal phase, and the field distribution at the output of the input coupler will be reproduced at the input of the output coupler. In the output star coupler, the light beams interfere constructively and converge at one single focal point on the focal line in the image plane. In this way, for the central

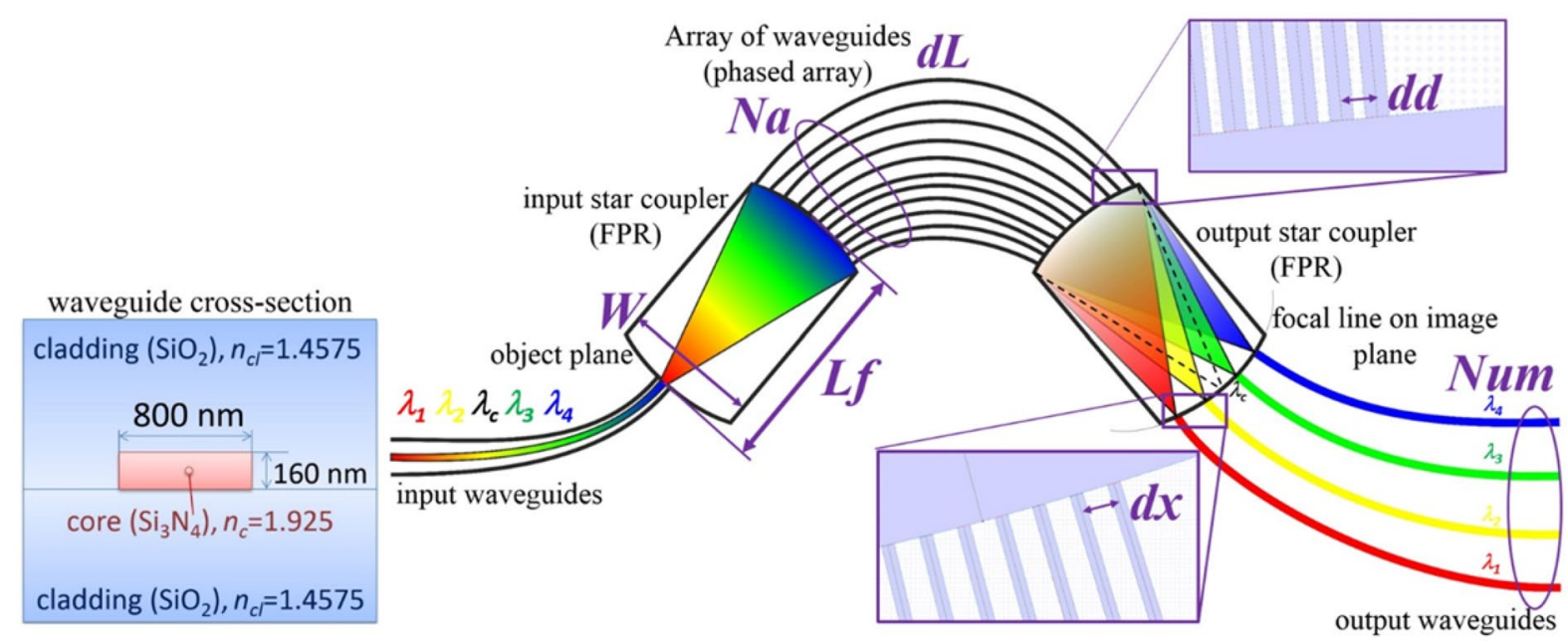

Fig. 1 Principle of an AWG with its design parameters and used waveguide cross-section 
wavelength $\lambda_{c}$, the input field at the object plane of the input star coupler is transferred to the center of the image plane of the output star coupler. If the wavelength is shifted to $\lambda_{c} \pm \Delta \lambda$ (i.e. $\lambda_{1}, \lambda_{2}, \ldots$ ), there will be a phase change in the individual PA waveguides that increases linearly from the lower to the upper channel. As a result, the phase front at the input aperture of the output star coupler will be slightly tilted, causing the beam to be focused on a different position in the image plane. The positioning of the output waveguides at the focal points in the image plane allows the spatial separation of the different wavelengths [8].

\section{AWG design}

All AWGs presented in this paper were designed for TMpolarized light with a central wavelength $\lambda_{c}=850 \mathrm{~nm}$. Figure 1 depicts the cross-section of the used waveguide geometry. The refractive index of the waveguide core at the center wavelength of $850 \mathrm{~nm}$ is $n_{\mathrm{c}}=1.925$ and that of the cladding $n_{\mathrm{cl}}=1.4575$. The waveguide size is $800 \mathrm{~nm} \times 160 \mathrm{~nm}$.

Every AWG design begins with the calculation of its dimensions, which are essential to create the AWG layout. The dimensions are given by the AWG design parameters, as shown in Fig. 1:

1. Minimum waveguide separation between PA waveguides (parameter $d d$ ),

2. Minimum waveguide separation between input/output waveguides (parameter $d x$ ),

3. Length of the star coupler (parameter $L f$ ), and.

4. Optical path length difference between adjacent waveguides in the phased array (parameter $d L$ ).
Width of the coupler $W$ is not a dominant parameter and can be freely changed. However, it should not be smaller than the width of the far-field intensity profile in the output aperture of the input star coupler. The number of array waveguides, $\mathrm{Na}$ is usually calculated from the width of the far-field intensity divided by the minimum waveguide separation between PA waveguides. In this way, all the light in the input star coupler is collected by the input array aperture [9]. Num is a number of output waveguides (transmitting channels) that the AWG is designed for.

For the calculations of the AWG design parameters, we used our in-house developed design tool called AWGParameters [9], which is based on [8]. The tool has been already applied for various AWG designs and is experimentally well proven [9]. We have started with a simple 8-channel, 100-GHz AWG design which calculated design parameters are as follows:

- Minimum waveguide separation between I/O waveguides: $d x(\mu \mathrm{m})=4$.

- Minimum waveguide separation between PA waveguides: $d d(\mu \mathrm{m})=1.2$.

- Coupler length: $L f(\mu \mathrm{m})=287.73$.

- PA waveguide length difference: $d L(\mu \mathrm{m})=58.9354$.

The calculated design parameters were then used as an input in the commercial photonics tool, WDM PHASAR from Optiwave [10], to create and to simulate the AWG structure. Figure 2 plots the simulated spectral response of this AWG together with the measured characteristics of the fabricated AWG.

Table 1 summarizes performance parameters calculated from the simulated/measured spectral responses together with the performance parameters that the AWG

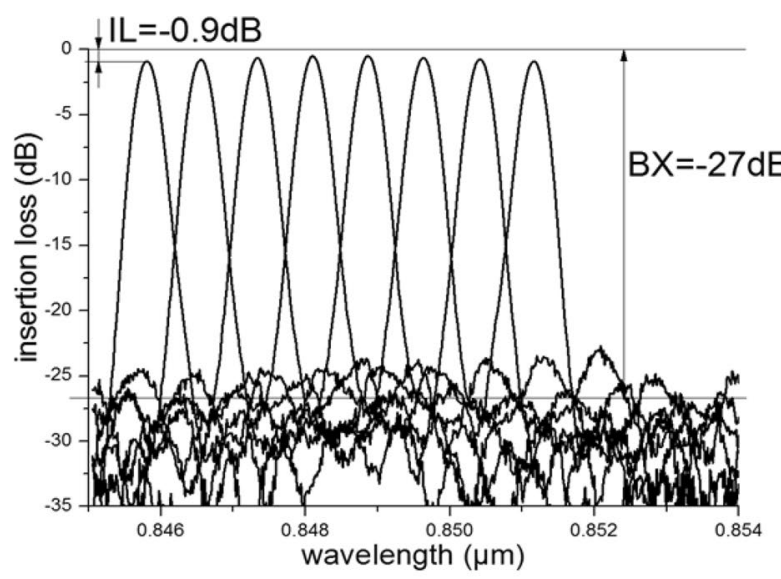

(a)

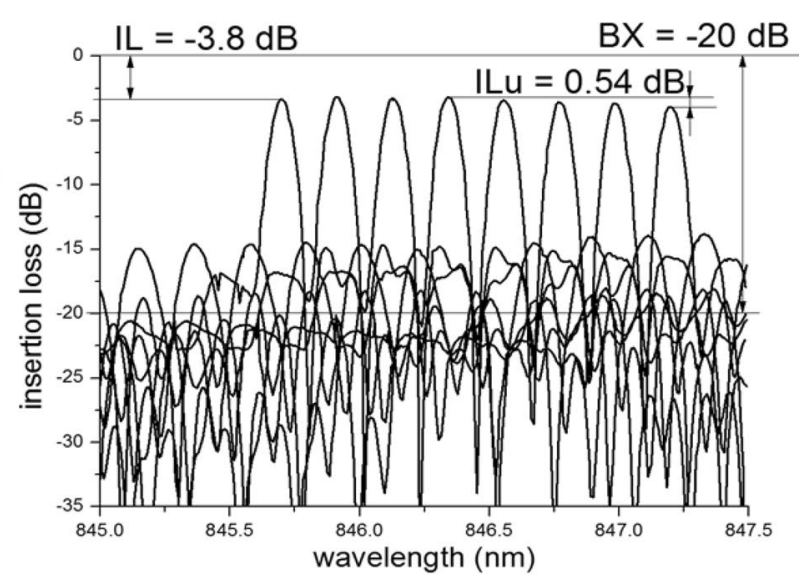

(b)

Fig. 2 Spectral response of the simulated 8-channel, 100-GHz AWG (a), together with the measured characteristics from fabricated AWG (b) 
Table 1 Performance parameters calculated from simulated/measured spectra together with the design parameters from AWGParameters tool

\begin{tabular}{llcc}
\hline & AWG-parameters & Simulation & Measurement \\
\hline Number of channels, Num & 8 & 8 & 8 \\
AWG central wavelength, $\lambda_{\mathrm{c}}$ & $850 \mathrm{~nm}$ & $848.5 \mathrm{~nm}$ & $846.46 \mathrm{~nm}$ \\
Channel spacing, Ch. Spacing & $100 \mathrm{GHz}$ & $100 \mathrm{GHz}$ & $100 \mathrm{GHz}$ \\
Insertion loss, IL & - & $-0.9 \mathrm{~dB}$ & $-3.8 \mathrm{~dB}$ \\
Insertion loss uniformity (non-uniform- & $0.4 \mathrm{~dB}$ & $0.42 \mathrm{~dB}$ & $0.54 \mathrm{~dB}$ \\
$\quad$ ity), ILu & $57.15 \mathrm{~dB}$ & $22.1 \mathrm{~dB}$ & $12.5 \mathrm{~dB}$ \\
Adjacent crosstalk, $A X$ & - & $-27 \mathrm{~dB}$ & $-20 \mathrm{~dB}$ \\
Background crosstalk, $B X$ & & &
\end{tabular}

was designed for. These parameters create the input for the calculations of AWG dimensions in AWG-Parameters tool.

\section{Design of standard 160-channel, $50-\mathrm{GHz}$ AWG-spectrometer}

In the next AWG designs, we have increased the number of the output channels (parameter Num) to 20, 40 and 80, respectively, and studied the influence of the design parameters (i.e. $d x, d d, L f, d L$ ) on the AWG performance. All these AWG designs were also technologically verified and feature satisfying optical properties [11-14]. Based on this study, we designed a standard 160-channel, 50-GHz AWG-spectrometer having a non-uniformity $I L u=1 \mathrm{~dB}$ [15]. The size of the AWG structure reached approximately $(1 \times 1.2) \mathrm{cm}^{2}$ (shown in Fig. 3a). In this design, the linear tapers were used.

Figure 4 depicts the corresponding simulated spectral response. As can be seen from Fig. 4b, the simulated nonuniformity $I L u$ of $1.26 \mathrm{~dB}$ is slightly higher than the design target $I L u=1 \mathrm{~dB}$. The insertion loss $I L$ reached $2.518 \mathrm{~dB}$, where $1.26 \mathrm{~dB}$ came from $I L u$.

On the other hand, the optical channels are well separated from each other. Figure 5 shows a detailed view of the spectral response of eleven channels at the beginning of the spectrum and of sixteen channels in the middle of the spectrum. An adjacent channel crosstalk of $A X=21.49 \mathrm{~dB}$ was calculated for that particular channel and it is the best case in the whole spectrum (Fig. 5a).

The adjacent channel crosstalk parameter in Fig. $5 \mathrm{~b}$ $(A X=18.2 \mathrm{~dB})$ is the worst case calculated over all

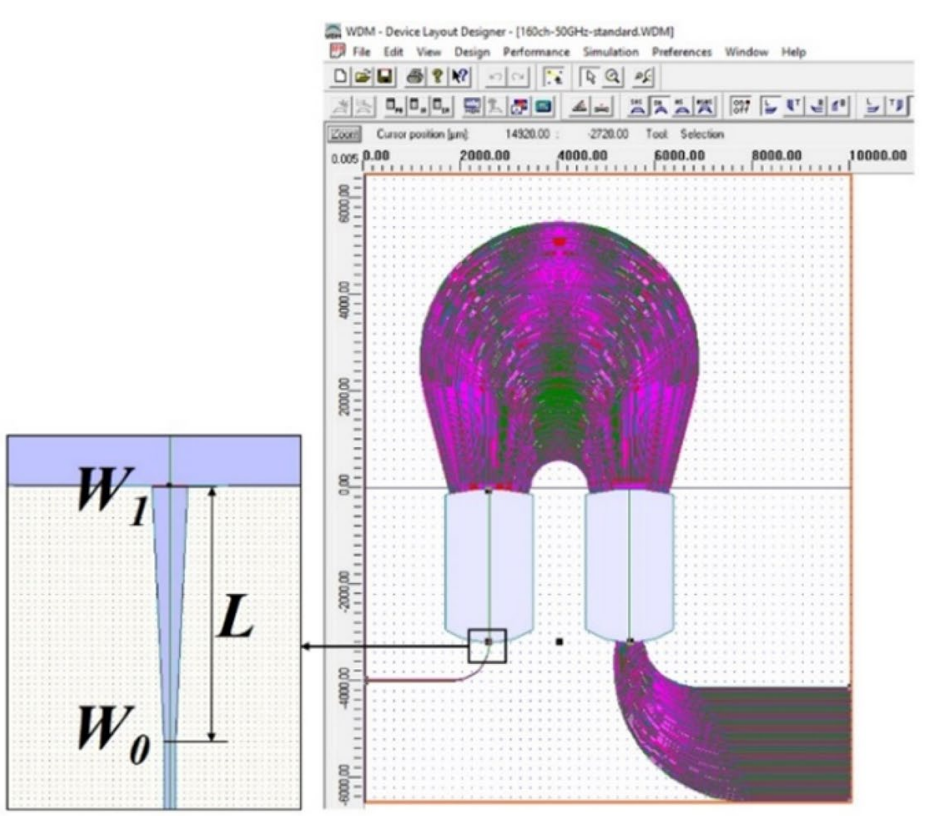

(a)

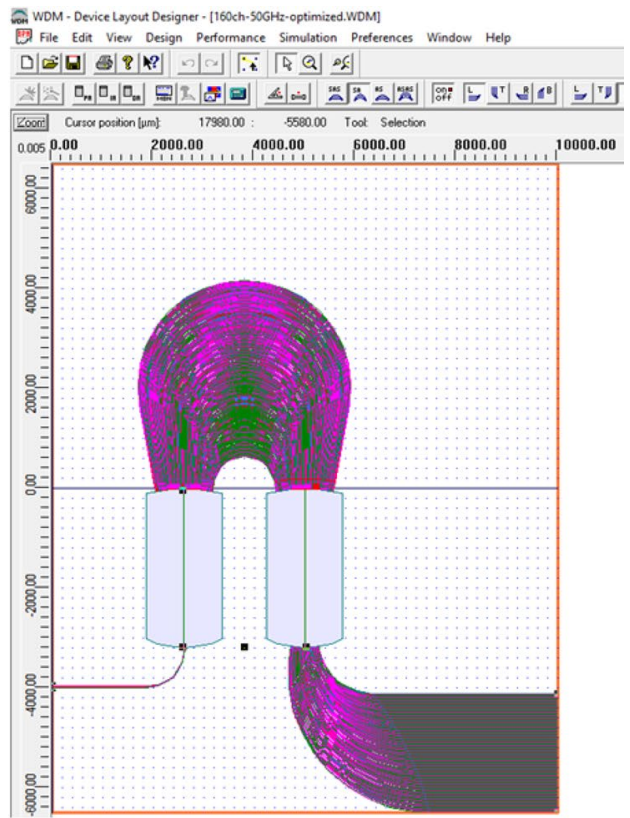

(b)

Fig. 3 Layout of $\mathrm{Si}_{3} \mathrm{~N}_{4}$-based 160-channel, 50-GHz AWG: a standard design with linear taper; $\mathbf{b}$ size-optimized design with specially shaped taper 


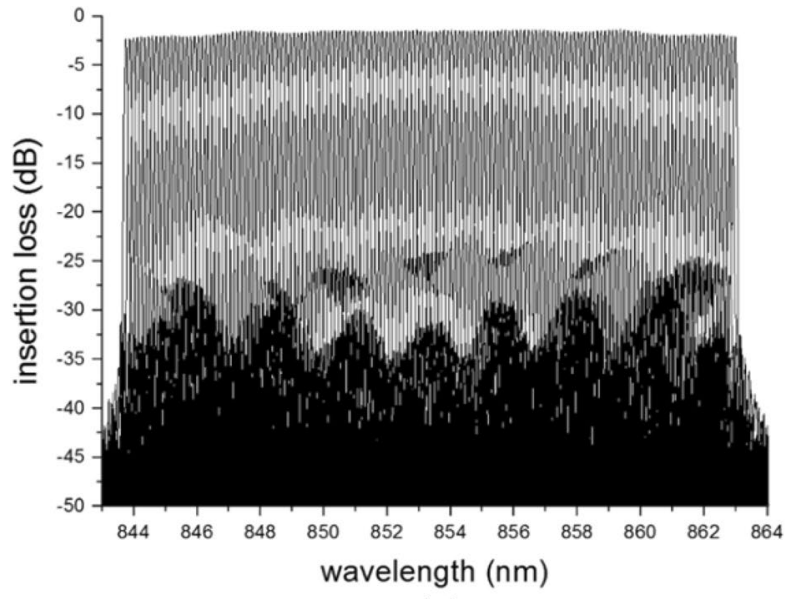

(a)

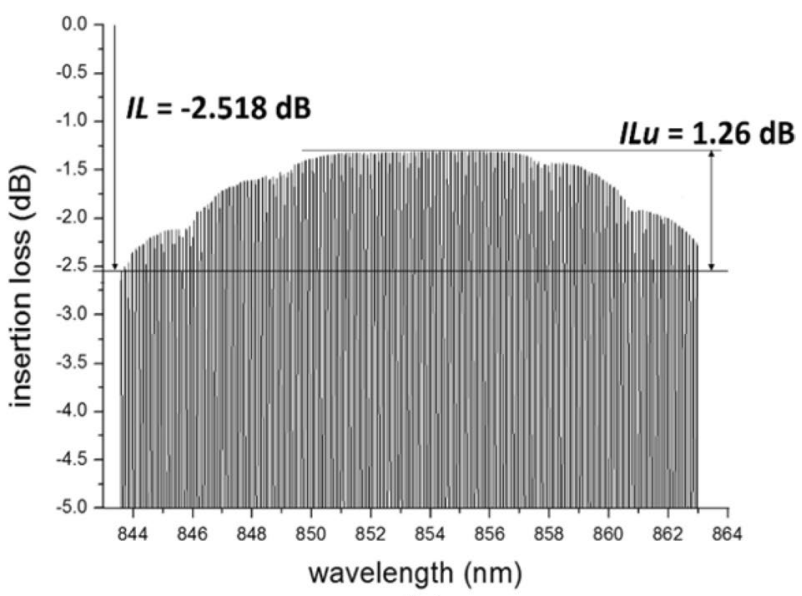

(b)

Fig. 4 a Simulated spectral response of standard 160-channel, 50-GHz AWG design applying linear taper, b detailed view of the insertion loss, $I L$ and insertion loss uniformity, $I L u$

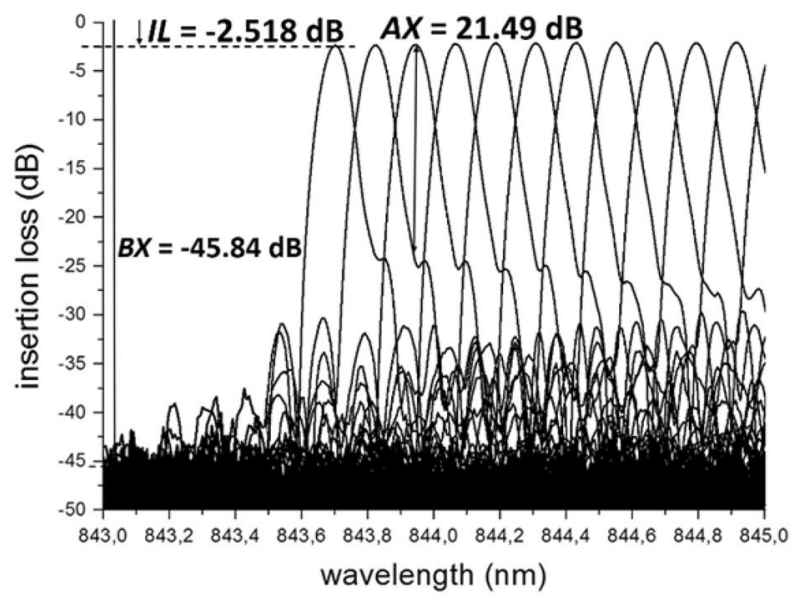

(a)

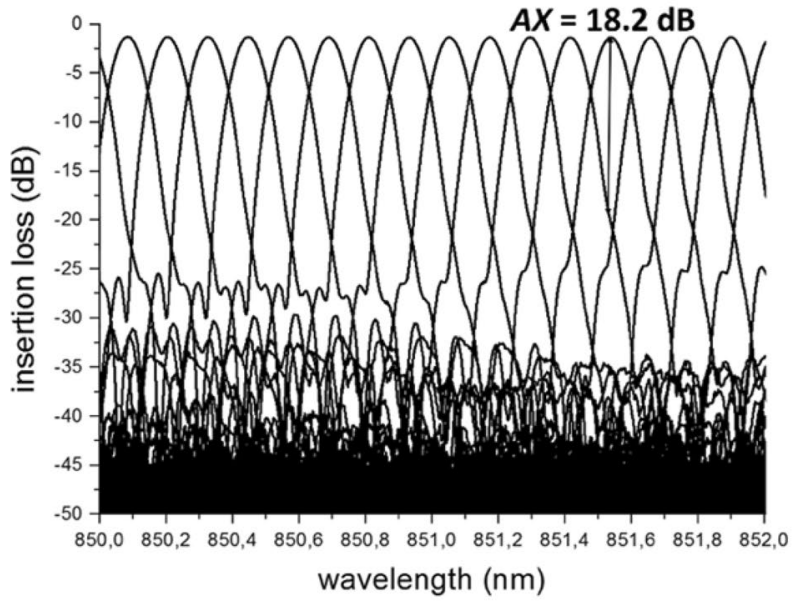

(b)

Fig. 5 Detailed view of the adjacent channel crosstalk of the standard 160-channel, 50-GHz AWG applying linear taper: a the best case, $\mathbf{b}$ the worst case calculated over all output channels

160 channels. The non-adjacent channel crosstalk reached $n A X=30.76 \mathrm{~dB}$ and the background crosstalk $B X=-45.84 \mathrm{~dB}$.

\section{Design of size-optimized 160-channel, 50-GHz AWG-spectrometer}

Arrayed waveguide gratings used as spectrometers usually require more than 160 output channels. In our work, we target AWG spectrometers with up to 1024 output channels. However, the size of such AWG structure grows to up to $(5 \times 5) \mathrm{cm}^{2}$. Therefore, one of the major tasks in the AWG development was the optimization of the structure size without affecting the optical performance of the designed AWG.

As described above, for the AWG design only a few parameters are necessary to calculate. The influence of the parameters $d d$ and $d x$ on the optical performance was tested in previous AWG designs and experimentally verified. Based on this, the values of $d d$ and $d x$ were fixed to keep losses and channel crosstalk low. The length of the coupler $(L f)$ is usually calculated to reach a particular uniformity, $I L u$ (in our case $I L u=1 \mathrm{~dB}$ ). The length difference between adjacent waveguides in the PA $(d L)$ is responsible for the focusing effect. It acts as a lens with image and object planes at a distance $L f$ from the array apertures, i.e., this parameter has to be calculated precisely. The width of the coupler $W$ 
should be set greater than the width of the far-field intensity in the star coupler to obtain sufficiently low crosstalk [8]. The number of array waveguides, $\mathrm{Na}$ is usually calculated from the width of the far-field intensity divided by the minimum waveguide separation between PA waveguides. From this follows, that to be able to reach a particular target performance, all AWG design parameters have to be set very carefully and, therefore, there is not much freedom in optimizing the AWG structure size. In our approach, we took the standard 160-channel, 50-GHz AWG structure described above (i.e. keeping the same design parameters $d d, d x, L f$, $d L)$ and placed a specially shaped taper in front of the input star coupler instead of the linear taper presented in Fig. 3a. Usually, such a taper may have a range of different shapes such as linear taper, multimode interference (MMI) coupler or parabolic waveguide horn taper, etc., to form the desired spectral response of the AWG, i.e., Gaussian shape, flat-top shape, or semiflat [16]. However, such tapers also determine the far-field intensity pattern. For example, changing the width of the linear taper ( $W_{1}$ in Fig. 3a) the width of the transmitted optical signal in the spectrum can be controlled (Wo usually equals the width of the waveguide). The wider the used linear taper the wider the optical signal and the narrower the far field intensity. However, the widening of the optical signals leads to a strong increase in the adjacent channel crosstalk. To prevent this crosstalk there is some limitation of the linear taper width, $W_{1}$. Our specially shaped taper has the same width, Wo and $W_{1}$ and the same length $L$ as the linear taper used in the standard AWG design, i.e. it forms a Gaussian shape very similar to the original one from Fig. 5 but with a narrower far-field intensity than the linear taper (more than 25\%, see Fig. 6) [17]. This means that the spectral response of the size-optimized 160-channel, 50-GHz AWG is similar to the standard 160-channel, 50-GHz AWG (as can be seen in Fig. 7, 8), but this design requires less PA waveguides, which reduces the size of the AWG structure by about 15\% (Fig. 3b).

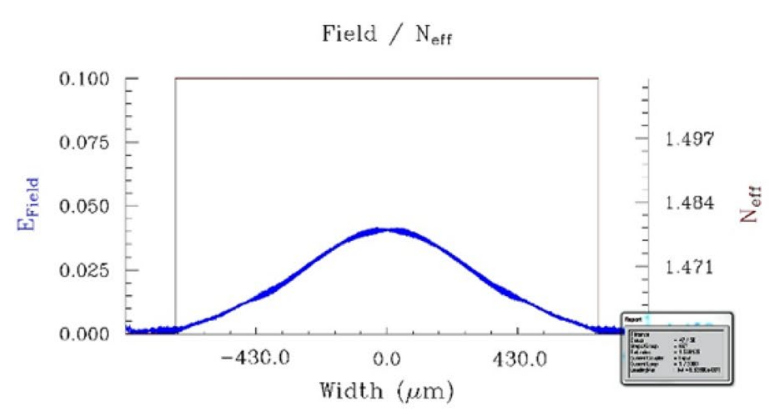

(a)

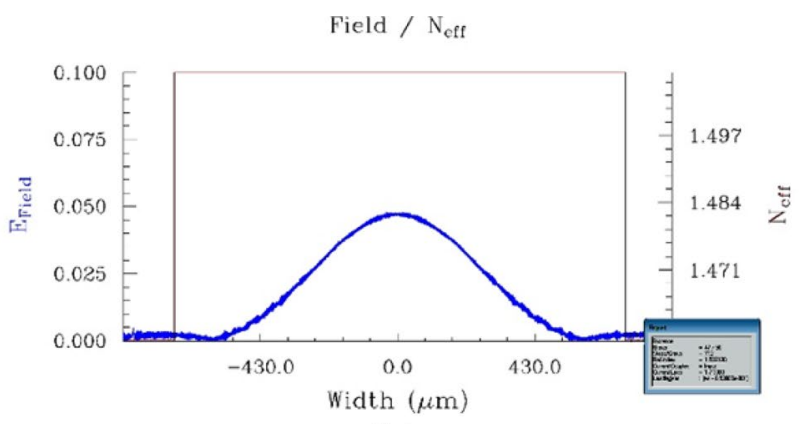

(b)

Fig. 6 Simulated far-field intensity in the input star coupler: a applying linear taper; $\mathbf{b}$ applying specially shaped taper

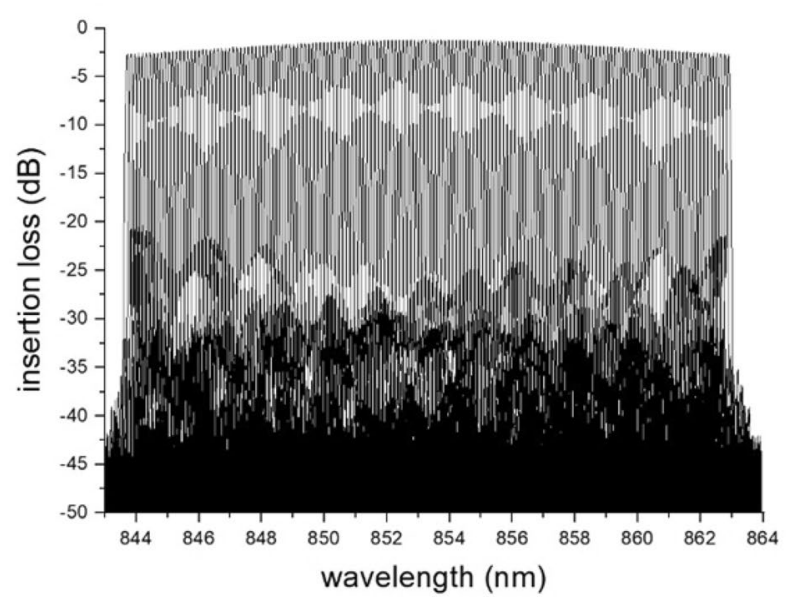

(a)

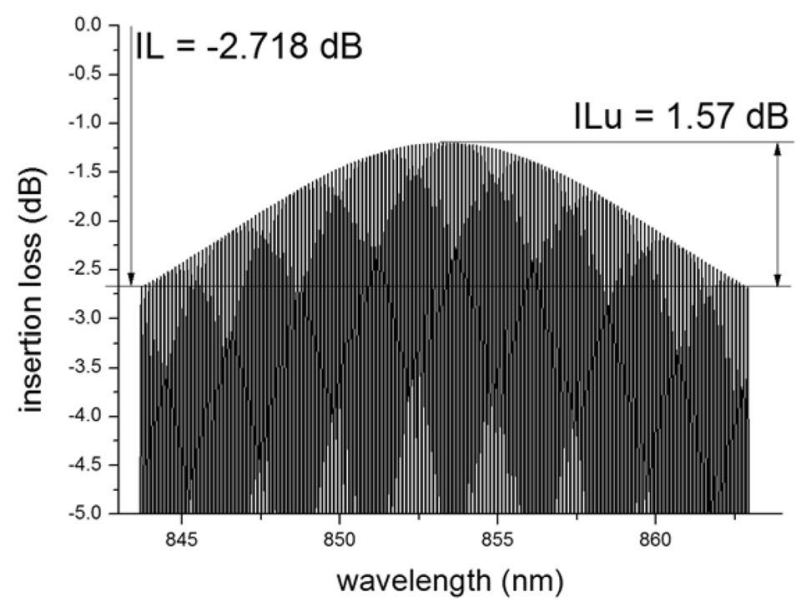

(b)

Fig. 7 a Simulated spectral response of size-optimized 160-channel, 50-GHz AWG design applying specially shaped taper, b detailed view of the insertion loss, $I L$ and insertion loss uniformity, $I L u$ 


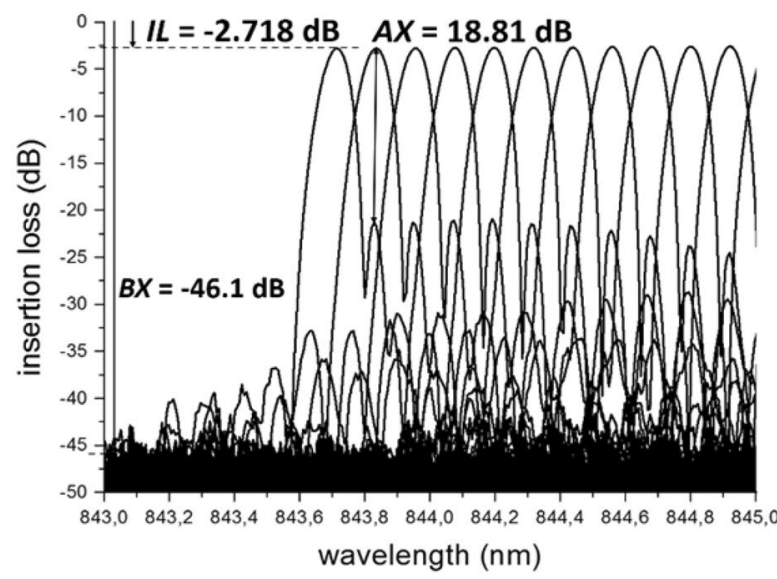

(a)

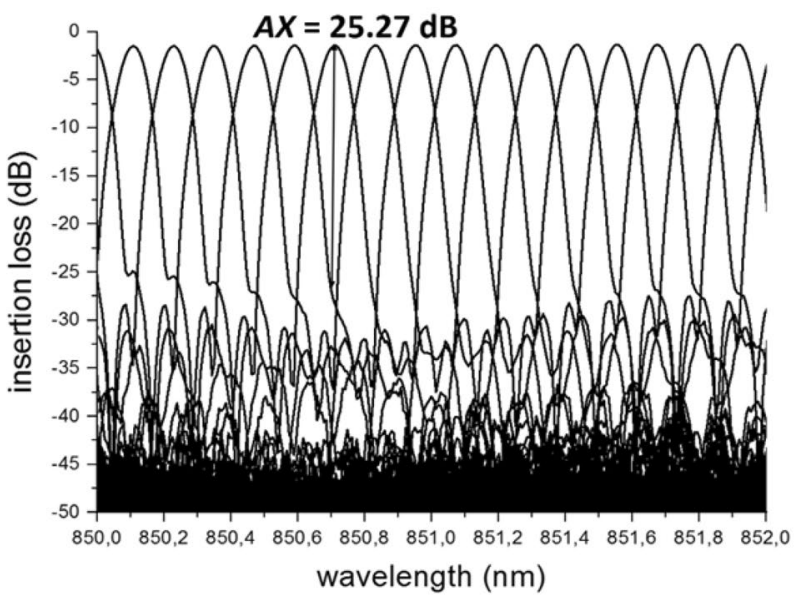

(b)

Fig. 8 Detailed view of the adjacent channel crosstalk $A X$ of the size-optimized 160-channel, 50-GHz AWG applying specially shaped taper: a the worst case; $\mathbf{b}$ the best case calculated over all output channels

In the size-optimized design, the simulated insertion loss and insertion loss uniformity reached slightly higher value $(I L=-2.718 \mathrm{~dB}$ and $I L u=1.57 \mathrm{~dB})$ compared to the standard design. On the other hand, the size-optimized spectrum features lower channel crosstalk compared to the standard design. The highest adjacent channel crosstalk was measured at the most outer channels (in Fig. 8a parameter $A X=18.81 \mathrm{~dB}$ ) and the lowest adjacent crosstalk in the middle of the spectrum (in Fig. $8 \mathrm{~b}$ parameter $A X=25.27 \mathrm{~dB}$ ). The non-adjacent channel crosstalk reached $n A X=31.948 \mathrm{~dB}$ and the background crosstalk $B X=-46.1 \mathrm{~dB}$.

\section{Technological verification}

Both AWG designs (standard and size-optimized) were fabricated at ams AG using standard CMOS processes. The process flow was as follows: a several $\mu \mathrm{m}$ thick $\mathrm{SiO}_{2}$ bottomcladding was deposited on a $\mathrm{Si}$ wafer before a $\mathrm{Si}_{3} \mathrm{~N}_{4}$ layer in the order of a few hundred nm was applied via PECVD at suitably low temperatures. This layer was structured by photolithography (180 nm technology) and subsequent etching of the SiN layer and, finally, covered with another few $\mu \mathrm{m}$ of $\mathrm{SiO}_{2}$ as top-cladding.

To obtain a smooth vertical surface for in- and out-coupling on the chip edges, a several dozen $\mu \mathrm{m}$ deep trench has been etched into the photonic layer stack and underlying $\mathrm{Si}$ around the chip edge before dicing. This trench allows for very efficient in-plane fiber coupling at both input and output facets, respectively. Additionally, inverted tapers were used for higher coupling-efficiency. All these processes are fully CMOS compatible and have been accompanied by standard measurements of the line widths before and after the $\mathrm{SiN}$ structuring using SEM, as well as thickness measurements of the different layers using ellipsometry. Figure 9a depicts the mask layout prepared for the fabrication and Fig. 9b shows the fabricated chip.

For the characterization of the optical transmission characteristics of the AWG we employed a SolsTiS Ti:Sapphire laser source [18], which can be tuned between a wavelength of 800-900 nm. The light was coupled into the chip from a polarization maintaining high numerical aperture (NA) fiber by means of an inverted taper with a tip width of $\sim(160 \times$ 160) $\mathrm{nm}^{2}$ at the chip edge. The coupling losses were of the order of $1.5 \mathrm{~dB}$ per coupling event. The propagation loss for the TM-like mode amounted to $\sim 0.5 \mathrm{~dB} / \mathrm{cm}$. At the individual output ports of the AWG, the light was collected with a standard single mode fiber. The transmitted power was measured with an optical power meter. For each channel the fiber was aligned to the corresponding output waveguide at the wavelength showing maximum power transmission. The alignment was optimized with a piezo-driven auto-alignment system to achieve optimum coupling. The obtained transmission loss was then normalized with the fiber-to-fiber transmission loss of a straight waveguide of $\sim 2 \mathrm{~cm}$ length to isolate the on-chip optical transmission characteristics of the AWG.

\section{Discussion}

The above described AWG designs were fabricated on five different wafers. Random samples were picked to measure the spectra. Figure 10 presents one of the measurement sets of both AWG designs (half spectrum of the standard AWG and full spectrum of the size-optimized AWG). When compared to standard design in Fig. 10a, the size-optimized 


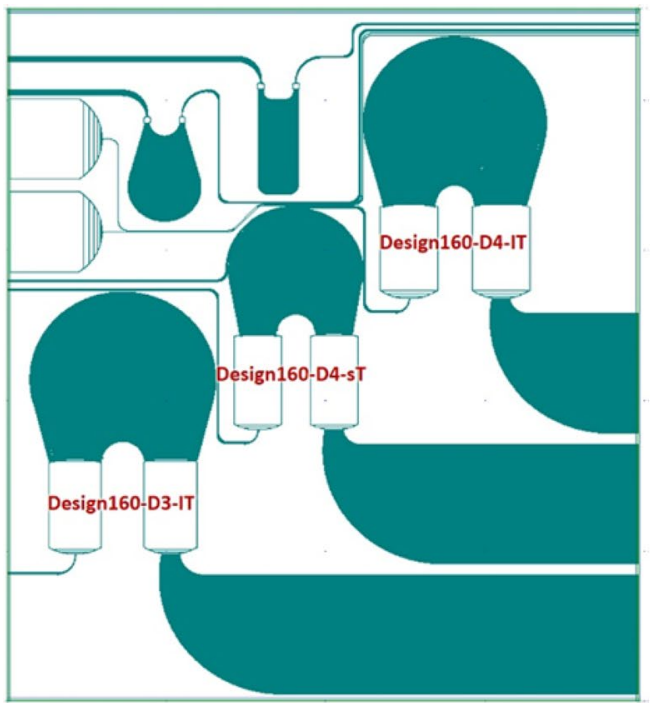

(a)

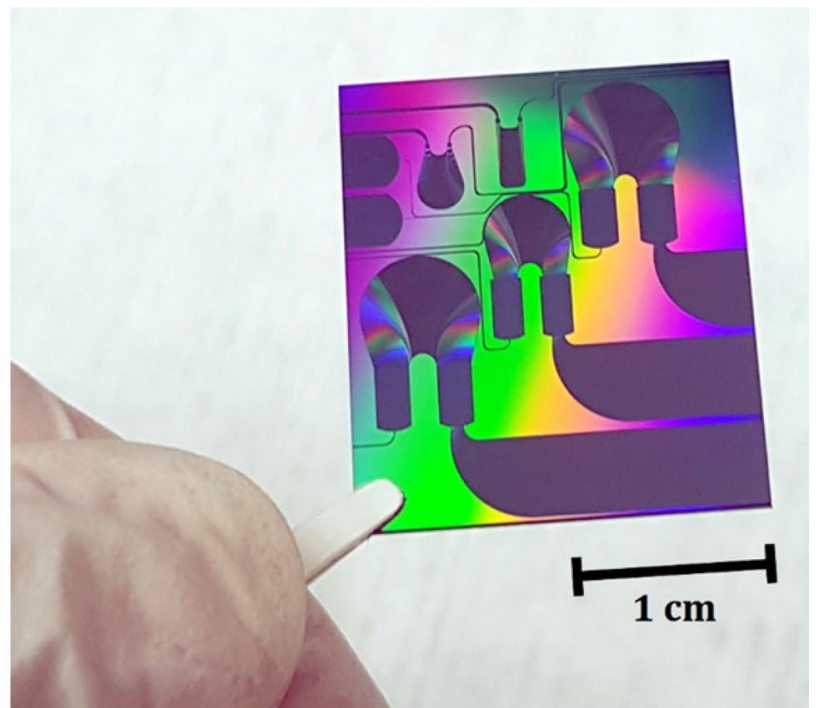

(b)

Fig. 9 a Mask layout with various 160-channel, 50-GHz AWG designs. Design "160-D4-1T" is a standard design with the linear taper; design "160-D4-sT" is a size-optimized design with the specially shaped taper. b Fabricated chip

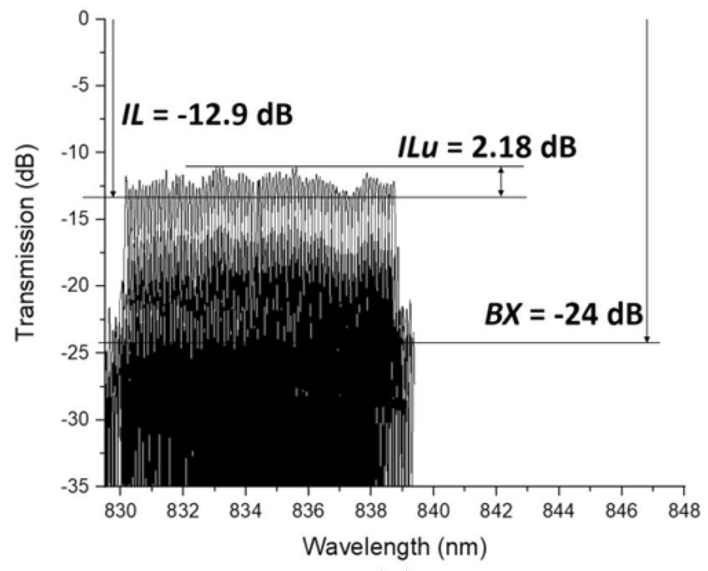

(a)

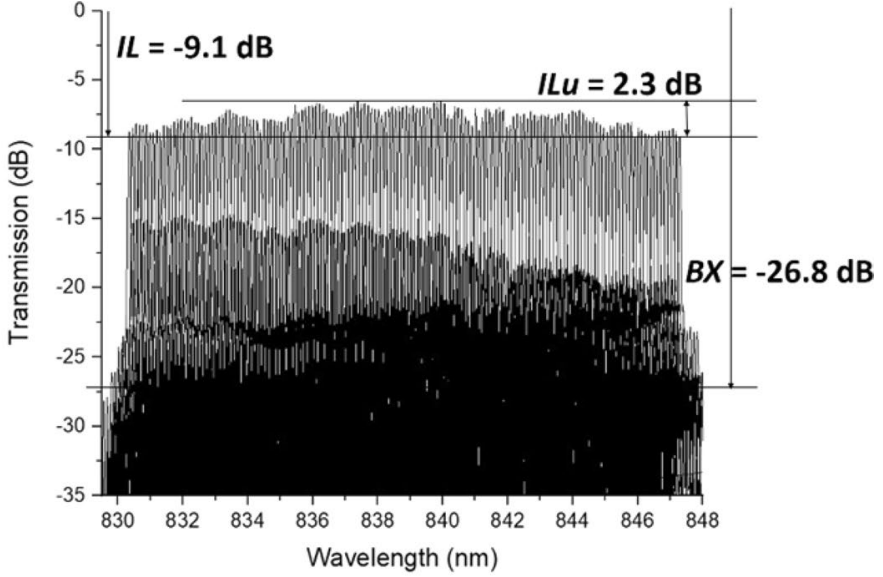

(b)

Fig. 10 Measured spectral responses of 160-channel, 50-GHz AWGs: standard design (a); size-optimized design (b)

AWG (Fig. 10b) reached very similar insertion loss uniformity, $I L u$ but considerably lower insertion loss $I L$. Its value reached $-9.1 \mathrm{~dB}$ that is $3.8 \mathrm{~dB}$ less than in the standard design where $I L=-12.9 \mathrm{~dB}$. We characterized all five sample sets and the measurement results confirm lower insertion loss in all spectra of the size-optimized AWG by more than $2 \mathrm{~dB}$ compared to the standard AWG. Additionally, the sizeoptimized AWG design features a lower background crosstalk of $B X=-26.8 \mathrm{~dB}$ compared to the standard design, where $B X$ reached $-24 \mathrm{~dB}$.

Adjacent channel crosstalks, calculated on the left side of both spectra (Fig. 11a), are similar to each other. Their values lie between 7 and $8 \mathrm{~dB}$. In the case of size-optimized
AWG design, the channel crosstalk on the right side of the spectrum reached considerably better value of $A X=12.7 \mathrm{~dB}$. Since in the case of the standard AWG only the left half of spectrum was measured, Fig. 11b shows the detailed view of the crosstalk calculated between the channels 79 and 80 in the middle of both spectra. As can be seen, the channels in the middle of the size-optimized spectrum are much better separated from each other $(A X=10 \mathrm{~dB})$ compared to the channels in the middle of the standard spectrum $(A X=5.7 \mathrm{~dB})$.

A 160-channel AWG was integrated in a standard fiberbased OCT system as depicted in Fig. 12. A SLD light source (Broad Lighter T840, Superlum) with a bandwidth 

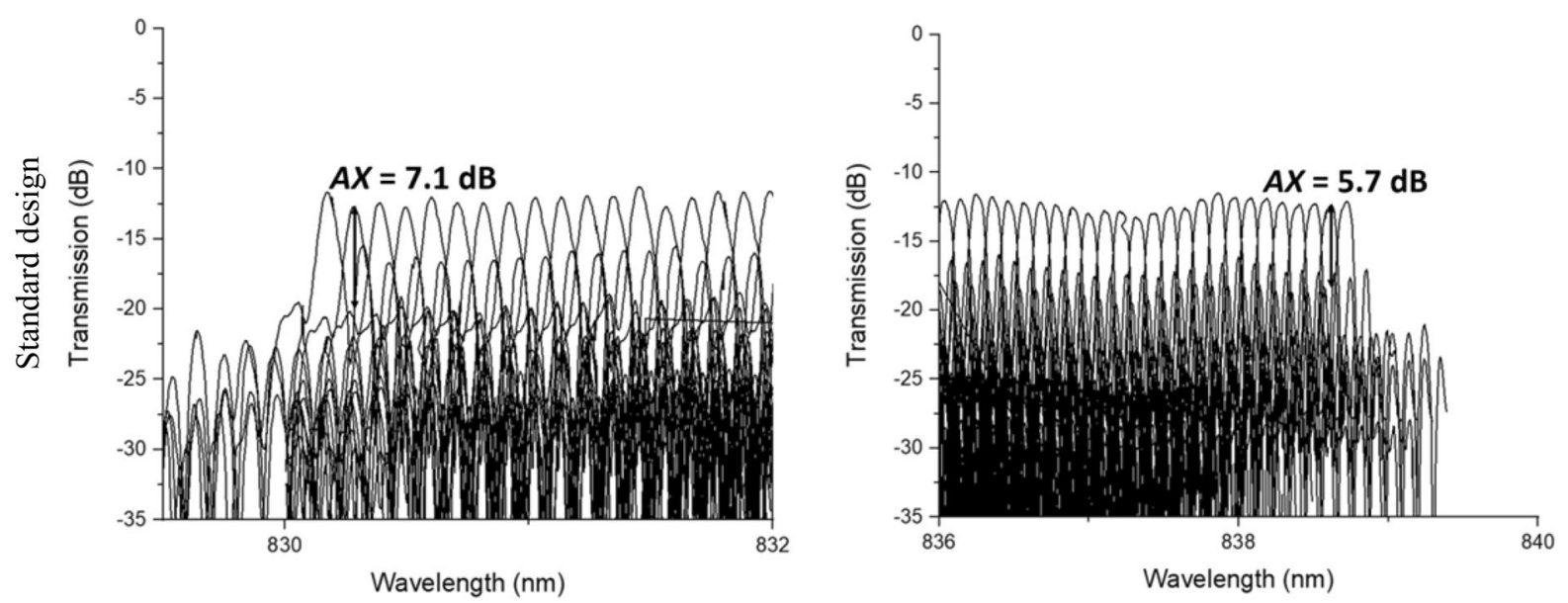

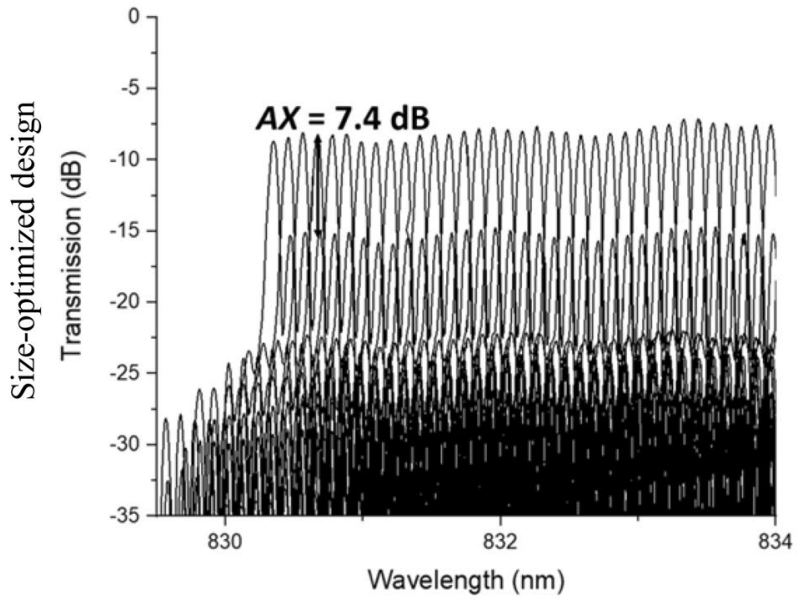

(a)

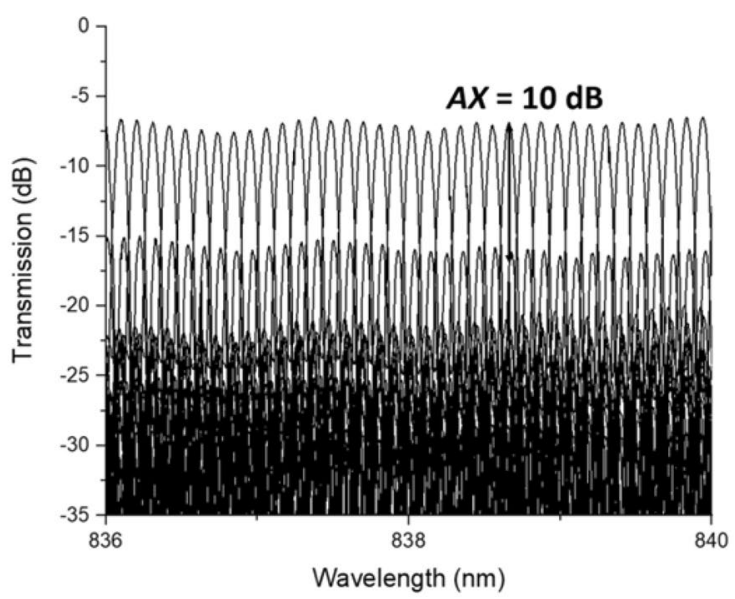

(b)

Fig. 11 Adjacent channel crosstalk calculated from detailed view of the measured spectra of standard and size-optimized AWGs: left side of the spectra (a); middle of the spectra (b)

of $820-850 \mathrm{~nm}$ was used to cover the bandwidth of the AWG. The interfered light was coupled in the AWG, using a single mode fiber. The individual channels were projected onto a CCD line camera (EV71 YEM4CL 2014BA9, AVIIVA). The FWHM of the intensity peak from a mirror represents the axial resolution of the system. With a measured axial resolution of $17 \mu \mathrm{m}$ in air (theoretically calculated: $15 \mu \mathrm{m}$ ) shows that all wavelengths are transmitted well. These parameters indicate that the 160-channel AWG can be used for OCT application and further integration will be performed.

The maximum imaging range was calculated via.

$z_{\max }=\frac{n_{0} \lambda c^{2}}{4 \delta_{s} \lambda}=\frac{1 \cdot(835 \mathrm{~nm})^{2}}{4 \cdot \frac{20 \mathrm{~nm}}{160 \text { channels }}}=1.49 \mathrm{~mm}$ in air,

which have to be confirmed in future measurements.

\section{Conclusion}

In this paper, we presented the design, simulation and technological verification of the size-reduction of a 160-channel, 50-GHz AWG-spectrometer. This design is based on a previous study of various AWG designs (8-channel, 100-GHz; 20-channel, 50-GHz; 40-channel, 50-GHz and 80-channel, 50-GHz AWGs), which were also experimentally well proven. The simulated results showed that applying a specially shaped taper in front of the input star coupler allows about $15 \%$ size-reduction of the device compared to a standard AWG structure while maintaining similar optical properties. All designed AWGs were fabricated and in total five samples were picked for characterization. Measured spectra show that, besides the size-reduction, the specially shaped taper also yields an improvement of the AWG performance in all cases. Particularly, the size-optimized AWG exhibits in 


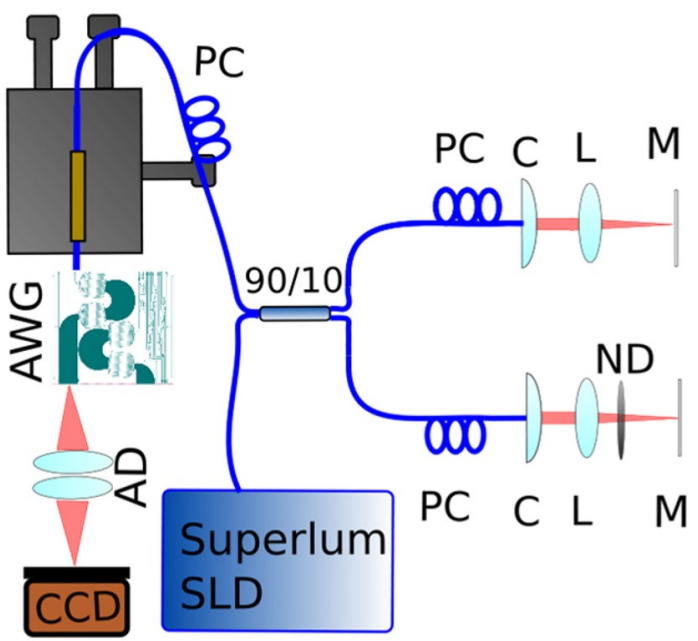

Fig. 12 A fiber-based OCT-system, integrating the 160-channel AWG. $10 \%$ of the light emitted by the light source is forwarded to the sample arm, $90 \%$ to the reference arm. Backscattered intensities interfere in the fiber coupler and is forwarded into the AWG by aligning a fiber to the input waveguide. An achromatic doublet pair (MAP103030-B) projects the individual channels on the CCD (PC polarization controller, $C$ fiber collimator, $L$ lens, $M$ mirror)

all spectra at least $2 \mathrm{~dB}$ lower insertion loss compared to the standard AWG design. Additionally, the channel crosstalk, calculated from both simulated and measured spectra, of the size-optimized AWG features also an improvement in comparison to the standard design.

Acknowledgements Open access funding provided by FH Vorarlberg-University of Applied Sciences. This work was carried out in the framework of the project COHESION, no. 848588, funded by the Austrian Research Promotion Agency (FFG).

Open Access This article is distributed under the terms of the Creative Commons Attribution 4.0 International License (http://creativeco mmons.org/licenses/by/4.0/), which permits unrestricted use, distribution, and reproduction in any medium, provided you give appropriate credit to the original author(s) and the source, provide a link to the Creative Commons license, and indicate if changes were made.

\section{References}

1. A. Kaneko et al., Design and applications of silica-based planar lightwave circuits. IEEE J. Sel. Topics Quantum Electron. 5, 1227-1236 (2002)

2. S. T. S. Cheung, et al. "Low-loss and high contrast silicon-oninsulator (SOI) arrayed waveguide grating," in Proc. Conf. Lasers Electro-Optics, pp. 1-2 (2012)

3. D. Dai et al., Low-loss $\mathrm{Si}_{3} \mathrm{~N}_{4}$ arrayed-waveguide grating (de) multiplexer using nano-core optical waveguides. Opt. Exp. 19, 14130-14136 (2011)
4. D. Martens et al., Compact silicon nitride arrayed waveguide gratings for very near-infrared wavelengths. IEEE Photonics Technol. Lett. 27, 137-140 (2015)

5. P. Cheben, "Wavelength dispersive planar waveguide devices: echelle gratings and arrayed waveguide gratings," Chapter 5 in Optical Waveguides: From Theory to Applied Technologies, M. L. Calvo and V. Lakshminarayanan, Eds., pp. 173-230, CRC Press, London (2007)

6. S.H. Kong et al., Integrated silicon microspectrometer. IEEE Instrum. Meas. Mag. 4, 34-38 (2001)

7. J.T. Bradshaw, S.B. Mendes, S.S. Saavedra, Planar integrated optical waveguide spectroscopy. Anal. Chem. 77, 28A-36A (2005)

8. M.K. Smit et al., PHASAR-based WDM-devices: principles, design and applications. J. Sel. Top. Quantum Electron. 2, 236250 (1996)

9. D. Seyringer, "Arrayed waveguide gratings," in SPIE Spotlights - New e-book series, SPIE Press, P.O. Box 10, Bellingham, Washington 98227-0010 USA

10. Optiwave: http://www.optiwave.com

11. D. Seyringer, et al. "Design and Simulation of $\mathrm{Si}_{3} \mathrm{~N}_{4}$ Based Arrayed Waveguide Gratings Applying AWG-Parameters Tool." Proc. of the 18th International Conference on Transparent Networks (ICTON 2016), 978-1-5090-1466-8/16, We.C5.5. (2016)

12. D. Seyringer, et al., "Design and simulation of 20-channel, 50-GHz Si3N4 based arrayed waveguide grating applying AWGParameters tool," Proc. SPIE Photonics West 2017, Integrated Optics: Devices, Materials, and Technologies XXI, Paper 101061L (2017)

13. D. Seyringer, C. Burtscher, L. Gajdosova, Si3N4 based 40-ch, 50-GHz AWG for medical applications (Proc, APCOM, 2017)

14. D. Seyringer, et al. "Design and Optimization of High-Channel Si3N4 based AWGs for Medical Applications," Proc. PHOTOPTICS 2018 (2018)

15. D. Seyringer, C. Burtscher, J. Edlinger, A. Maese-Novo, P. Muellner, R. Hainberger, J. Kraft, G. Koppitsch, G. Meinhardt, and M. Sagmeister, "Design of 160-Channel $\mathrm{Si}_{3} \mathrm{~N}_{4}$ based AWGSpectrometer for Medical Applications," Proc. 2018 TechConnect World Innovation Conference and Expo (2018)

16. E. Wildermuth, "Performance optimization of flat-top passband arrayed waveguide grating demultiplexers," Dissertation ETH. Zürich, No. 13440 (1999)

17. D. Seyringer, C. Burtscher, J. Edlinger, W. Drexler, E. Rank, P. Muellner, R. Hainberger, A. Maese-Novo, M. Vlaskovic, H. Zimmermann, J. Kraft, G. Koppitsch, M. Sagmeister, G. Meinhardt, "Size reduction of high-channel Si3N4 based AWG-spectrometer for medical applications", SPIE Nanoscience + Engineering, 2018, San Diego, California, United States; Proceedings Volume 10730, Nanoengineering: Fabrication, Properties, Optics, and Devices XV; 107300R (2018), https://doi.org/10.1117/12.2319670

18. M Squared, 1 Kelvin Campus, West of Scotland Science Park, Glasgow G20 0SP, UK

Publisher's Note Springer Nature remains neutral with regard to jurisdictional claims in published maps and institutional affiliations. 